\title{
Efectos de la estructura \\ productiva en el crecimiento económico de las ciudades de la región centro de México durante el periodo $1998-2013^{*}$
}

Effects of the productive structure on the economic growth of cities

in the central region of Mexico during the period 1998-2013

Josué Gómez Castellanos***

Recibido: 30 de marzo de 2020

Revisado: 20 de abril de 2020

Aprobado: 30 de abril de 2020 


\section{Resumen}

En este artículo se analiza la estructura productiva de las ciudades de la región centro de México y su influencia en el crecimiento económico local entre 1998 y 2013. Se estudian las catorce ciudades de la región centro de más de 100000 habitantes según el Catálogo del Sistema Urbano Nacional 2012. Los resultados muestran que las ciudades de menor tamaño se especializan en sectores de valor agregado bajo, mientras que las de mayor tamaño han mantenido su especialización en sectores industriales modernos y en servicios de alto valor agregado con tendencia a la diversificación. Ante ello, el crecimiento económico local ha sido impulsado principalmente por la diversificación productiva y por algunos sectores industriales de alto valor, lo cual ha determinado ritmos de crecimiento diferenciales entre ciudades.

Palabras clave: crecimiento económico, especialización, diversificación.

Clasificación JEL: O47, L16, L25.

\section{Abstract}

This article analyses the productive structure of the cities in Central Mexico and its influence in local economic growth from 1998 to 2013. This research focuses on fourteen cities with more than a hundred thousand inhabitants according to the National Urban System 2012 catalogue. The results show that smaller cities are specialized in low value-added sectors, whereas larger cities have maintained their specialization in modern industrial sectors as well as high value-added services that tend towards diversification. Thus, local economic growth has been propelled by productive diversification and some high valueadded industrial sectors, which has caused differential growth rates among cities.

Keywords: economic growth, specialization, diversification.

JEL Classification: O47, L16, L25. 


\section{Introducción}

La región central de México ha sido, desde tiempos prehispánicos, una de las zonas más importantes del país, en la que se instalaron grandes asentamientos y actualmente es la más poblada del país (Negrete, 2008). Esta región fue el polo de desarrollo industrial durante el siglo xx, lo que condujo a una fuerte migración del campo hacia la Ciudad de México, capital del país y la ciudad más importante en términos demográficos y económicos. Esto ocasionó que el centro del país llegara a concentrar el 33\% de la población total en 1990 (Aguilar, 1999).

Durante la década de 1980, la región experimentó una transformación de su estructura económica, derivada de la trasferencia de capital y fuerza de trabajo desde los sectores industriales hacia los sectores de servicios que comenzaron a predominar en las grandes metrópolis (Graizbord \& Ruiz, 1999; Valdivia, 2008). Esta situación trajo consigo procesos simultáneos de concentración y descentralización de actividades productivas entre las ciudades de la región, lo que modificó los patrones de especialización y diversificación productiva del sistema urbano. Los efectos de la nueva configuración productiva en algunos casos atenuaron las desigualdades preexistentes entre ciudades (Garrocho, 2015), mientras que en otros posibilitaron la emergencia de ciudades medias vibrantes.

La reconfiguración de la estructura productiva del sistema urbano en la región ha tenido efectos sustanciales en el crecimiento económico de las ciudades, algunas de las cuales se han beneficiado de la descentralización de ciertas actividades de las metrópolis; otras han perdido dinamismo ante el surgimiento de ciudades más dinámicas (González \& Peyrelongue, 1990; Valdivia, 2008). De esta manera, en la región se han presentado dos tendencias contrapuestas: mientras que los sectores de servicios tienden a la concentración, los sectores industriales se dispersan entre ciudades de menor tamaño, con lo cual surge una forma urbana policéntrica conformada por un pequeño cúmulo de ciudades especializadas, formando un patrón complejo de encadenamientos espaciales y productivos (Aguilar, 2002).

La articulación y crecimiento entre ciudades deriva principalmente de su capacidad para integrarse en la estructura urbana regional. Su estructura económica, ya sea especializada o diversificada, es uno de los rasgos más importante que le permite articularse con otras ciudades (Aguilar \& Vásquez, 2000). Las ciudades más grandes se benefician de una mayor variedad productiva, lo cual genera desventajas para las ciudades medias y pequeñas que tienen que buscar actividades en las que puedan competir, ocasionando un crecimiento desigual entre ciudades. Por lo tanto, la identificación de la especialización o diversificación productiva puede permitir la generación de estrategias para fortalecer e incentivar sectores productivos en ciudades, con el fin de mejorar el crecimiento económico local y la interrelación del sistema urbano-regional. 
El objetivo de este trabajo es analizar la estructura productiva de las ciudades de la región centro de México y su influencia en el crecimiento económico local entre 1998 y 2013. El documento se estructura en cinco apartados, después de esta introducción. En el primero se exponen las principales teorías relacionadas con el crecimiento económico local, así como su relación con la estructura productiva. En el segundo se trata brevemente la metodología utilizada y las fuentes de información. En el tercero se analiza la estructura productiva de las ciudades. En el cuarto se estudia el crecimiento económico y su relación con la estructura productiva. Finalmente, en el quinto se establecen las conclusiones, señalando los principales hallazgos, así como la agenda de investigación.

\section{Crecimiento económico local y estructura productiva}

El crecimiento económico local se ha posicionado como uno de los temas más importantes de las últimas décadas, ya que las ciudades representan el motor de los países (Bluestone, Stevenson, \& Williams, 2008). Las metrópolis son los sitios donde la riqueza se genera y su crecimiento depende de las actividades productivas que se establecen en sus territorios. La actividad y el crecimiento económico no están aglutinadas sobre la cabeza de un alfiler, están localizadas desigual y temporalmente entre los territorios (Fujita \& Thisse, 2013). Por ello, el crecimiento local es un fenómeno altamente complejo con explicaciones que pueden variar entre regiones, países o continentes (Balchin, Isaac, \& Chen, 2000). Tomando en consideración estos factores, emergió la llamada geografía económica, como una rama de la ciencia económica avocada a estudiar la organización espacial de los sistemas económicos: dónde están localizadas las actividades productivas, cómo se conectan y cuáles son las consecuencias espaciales de los fenómenos económi$\cos$ (Dicken, 2011).

Dentro de la geografía económica se han desarrollado distintos enfoques y teorías para tratar de explicar la influencia del espacio en el crecimiento. Una de las primeras fue la teoría de la causación acumulativa, desarrollada como una teoría general de la concentración económica y el crecimiento autosustentado, que tenía sus bases en los efectos multiplicadores y las ventajas iniciales (Dicken \& Lloyd, 1990; Mills \& McDonald, 1992). Estos planteamientos fueron retomados años más tarde para dar origen al denominado modelo centro-periferia, en el cual se incorporaron supuestos como la competencia interregional desigual y la contraposición entre un sector económico moderno y uno tradicional (Richardson, 1978).

Otras de las teorías gestadas en la economía urbana se han fundamentado en las llamadas economías de localización y economías de aglomeración, que producen beneficios por la concentración de actividades productivas en un mismo lugar (Glaeser, 2008). Este 
tipo de economías también se conocen como economías externas, una de las mayores fuentes de explicación para la concentración espacial de las actividades económicas (Sobrino, 2003). La consideración de las economías de aglomeración y localización ha sido un elemento esencial para explicar el tamaño y distribución de las urbes modernas, así como para entender su crecimiento (Nakamura \& Morrison, 2006).

En décadas recientes surgió la denominada nueva geografía económica, que se fundamenta en los trabajos clásicos de la teoría de la localización. Esta teoría es una combinación de la ventaja comparativa y la noción del espacio en los procesos económicos. Trata de explicar la concentración productiva a partir de la interacción entre fuerzas centrípetas, que incentivan a aglutinar los sectores, y fuerzas centrífugas, que ocasionan la dispersión de la actividad (Krugman \& Fujita, 2004). El resultado de la contraposición de ambas fuerzas dependerá de los diferentes atributos espaciales, con lo cual se podrán suscitar procesos de reestructuración económica funcional al interior de regiones o países (Asuad, 2012). El balance entre beneficios y pérdidas determina la reubicación o contención de sectores productivos, pues la naturaleza espacial del proceso económico genera frenos ante cualquier proceso explosivo, ya que implicaría el sobreconsumo del espacio físico que deriva generalmente en congestión (McCann, 2001).

Las teorías de la concentración y dispersión de actividades económicas se han aplicado para el estudio del crecimiento económico local. Cingano y Schivardi (2004) mencionan que, con base en los planteamientos que realizó Marshall en 1890 sobre la importancia de la presencia de economías locales para la concentración económica, se han elaborado postulados teóricos alternativos para explicar los efectos de la intensidad y la composición sectorial productiva en el crecimiento económico local. Uno de los trabajos más importantes en el estudio de la especialización sectorial es la investigación realizada por Glaser, Kallal, Scheiman y Shleifer (1992), en el que establecen que la especialización productiva puede dar origen a economías de localización. Por su parte, la diversificación productiva puede generar economías de urbanización. Tanto una como otra pueden impulsar el crecimiento económico dependiendo de su fuerza y prevalencia.

La especialización y diversificación productiva son concepciones para explicar la composición económica de países, regiones o ciudades. La especialización se produce cuando un territorio destina la mayor parte de empleo o producción a uno o pocos sectores. Por contraparte, la diversificación se genera por el hecho de tener una amplia gama de actividades en las que está distribuida la economía del lugar. La especialización también se ha definido como el grado al cual la producción de una ciudad se encuentra dominada por alguna o algunas actividades relacionadas cercanamente (Bostic, Gans, \& Stern, 1997). También se le identifica como una composición industrial de una zona específica, en la que algunas industrias están aglomeradas en comparación con sus contrapartes nacionales (Nakamura \& Morrison, 2006). 
Los efectos de la especialización en el crecimiento económico local han sido evidenciados en diversos estudios. De acuerdo con Mokyr (1990), la especialización conlleva beneficios para el crecimiento económico derivado del progreso tecnológico que esta implica. En el estudio se señala que esto ha sido explicado desde los planteamientos de Adam Smith, pues se pensaba que el avance tecnológico era necesariamente un resultado de la especialización. Para Glaser et al. (1992), la especialización tiene un efecto positivo en el crecimiento, tanto en el de la propia industria como en el de la región donde se localiza, ya que las externalidades tecnológicas se generan en el seno de la industria.

Una composición productiva especializada puede generar un mayor beneficio de las economías de escala. Sin embargo, esto está sujeto al tamaño del mercado, que se encuentra determinado por la densidad y cantidad de población, así como por las restricciones de los costos de transporte (Beeson, 1992). Los beneficios en el crecimiento local dependerán de la capacidad para poder aprovechar las economías de escala. Por lo tanto, el tamaño de la ciudad, así como la especialización en un sector vibrante, podrá dar pauta a un crecimiento importante.

Como se ha visto, hay evidencia a favor de los efectos positivos de la especialización productiva. No obstante, también se han realizado estudios que demuestran que la especialización puede generar riesgos al crecimiento y que es la diversificación productiva la que conlleva el crecimiento de las ciudades por efectos de las economías de urbanización. Jacobs (1984) critica en su obra la idea de Adam Smith acerca de que la especialización genera eficiencia dentro del proceso de producción, ya que sostuvo que para este la eficiencia aparece al mismo tiempo como un resultado y como una causa; además, el hecho de que algunos trabajadores se encuentren enfocados en ciertas actividades no conduce necesariamente a una mayor eficiencia productiva.

Quigley (1998) establece que, derivado de los postulados de Marshall (1890), la diversificación y el tamaño impulsan el crecimiento por los efectos de las economías de escala, ya que la ampliación de una producción más heterogénea genera ahorros en costos, principalmente de insumos, transporte y, sobre todo, de transacciones. Aunado a esto, Duranton y Puga (2013) señalan que la diversificación puede facilitar el proceso de aprendizaje de las unidades de producción, dado que cuentan con una amplia gama de productos y servicios, de tal manera que pueden generar innovaciones más rápidamente.

\section{Metodología}

El presente estudio se realiza a escala de ciudad, por lo que comprende las ciudades de la región central de México con más de 100000 habitantes en 2010 según el Catálogo del Sistema Urbano Nacional 2012 del Consejo Nacional de Población (Conapo). 
De esta manera, se analizan las catorce ciudades más importantes de la región. En esta clasificación, las ciudades se componen de diversos municipios, ya sea que se trate de una zona metropolitana, una conurbación o de un solo municipio. Esta unidad de análisis permite estudiar las metrópolis como un todo, pues las áreas metropolitanas están muy cercanas a aproximar el mercado de trabajo y son las unidades más relevantes para expresar recursos (Huallacháin, 1992).

El análisis de estructura productiva y crecimiento económico a escala de ciudad implica algunas dificultades, ya que no se cuenta con información de Producto Interno Bruto (PIB) a este nivel de análisis. La información disponible comprende el valor bruto de la producción, valor agregado censal bruto y personal ocupado total por sector y municipio. Por lo tanto, para estimar la actividad económica y el crecimiento se utilizará el personal ocupado total agregado a escala de ciudad. La información fue obtenida de los Censos Económicos del Instituto Nacional de Estadística y Geografía (INEGI) para los años de 1999, 2004, 2009 y 2014.

El estudio se realizó generando una clasificación de sectores según su valor agregado (vA), lo cual requirió crear una agrupación con base en las remuneraciones promedio y el grado tecnológico. De esta manera, se generaron cuatro grandes clasificaciones: industria de va bajo, industria de va alto, servicios de va bajo y servicios de va alto. La agrupación se realizó utilizando criterios combinados de Molero e Hidalgo (2007) y Álvarez y Guimón (2010); además, la clasificación completa se puede consultar en el anexo 1.

Para analizar la estructura productiva, se utiliza el cociente de localización como medida de especialización tal como lo realizan Glaser et al. (1992) y Fu, Dong y Chai (2010). Dicho cociente se calcula a partir de personal ocupado con la siguiente fórmula:

$$
L Q_{i j}=\frac{x_{i j} / x_{* j}}{x_{i^{*}} / x_{* *}}
$$

Dónde $x_{i j}$ es el empleo en el sector $i$ en la ciudad $j ; x_{* j}$ es el empleo total en la ciudad $j$; $x_{i^{*}}$ es el empleo total del sector $i$ en la región, y $x_{* *}$ es el empleo total en la región. Este índice estima la especialización del sector $i$ en la ciudad $j$ relativo a la especialización del sector $i$ en toda la región. Un valor mayor a 1 indica que la ciudad $j$ está especializada en el sector $i$, es decir, que la ciudad $j$ ocupa una mayor proporción de su empleo en dicho sector que la proporción que se emplea a escala regional.

Para estimar el grado de diversificación económica se emplea el índice de diversificación absoluta, que utiliza información de personal ocupado y que se calcula de la siguiente manera:

$$
I D A_{j}=\frac{1}{\sum_{i}^{n}\left(x_{i j} / x_{* j}\right)^{2}}
$$


Dónde $x_{i j}$ es el empleo en el sector $i$ en la ciudad $j ; x_{x_{j}}$ es el empleo total en la ciudad $j$. Los valores del índice van desde 1, que indica una estructura productiva concentrada, a un valor infinito, que indica una estructura productiva diversificada. Cuanto mayor sea el valor del índice más diversificada se encuentra la ciudad.

\section{Características generales y estructura productiva de las ciudades}

En términos de población, las 14 ciudades que se estudian albergan la mayor parte de la población de la región: alrededor de 31 millones en 2015, que representa el $79 \%$. El Valle de México es la ciudad más importante que concentra más del $50 \%$ de la población regional, con más de 20 millones de habitantes. Esta es una zona metropolitana que está integrada por 76 municipios pertenecientes a tres estados de la república: 16 pertenecen a la Ciudad de México, 59 al estado de México y 1 a Hidalgo. El resto de ciudades cuentan con una población por debajo de los tres millones de habitantes, lo que muestra la gran desigualdad entre urbes de la región. Dichas ciudades se encuentran localizadas, en su mayor parte, alrededor del Valle de México, en lo que se ha denominado corona regional. El resto de ciudades se localiza en una periferia más lejana a la capital del país, que las hace funcionar como nodos articuladores con las demás regiones del país.

En cuanto a actividad económica, al igual que en términos de población, las ciudades en estudio tienen una importancia primordial. Nuevamente el Valle de México es la ciudad con la mayor concentración económica, pues aporta casi más del $50 \%$ de la actividad regional. La participación de las ciudades se acentúa o disminuye ligeramente según se trate del tipo de sector que se analice, lo cual refleja los cambios en la composición productiva.

Los resultados del análisis de la especialización productiva en las ciudades de la región centro muestran que hay una fuerte especialización en la industria de va bajo. De las catorce ciudades en estudio, diez se encuentran especializadas en este tipo de sectores, las cuales se localizan en prácticamente todo el ancho de la región (figura 1). Las ciudades con mayor grado de especialización en este tipo de sector son Teziutlán y San Juan del Río, las cuales se encuentran alejadas de la ciudad principal de la región y cuentan con una población relativamente pequeña. Otras de las ciudades que mostraron un avance en la especialización de este sector son Toluca y Puebla-Tlaxcala. Sin embargo, Toluca fue la única que evidenció un incremento significativo de su grado de concentración de empleo en esta industria. El resto de ciudades mencionadas anteriormente no reportaron aumentos importantes de su nivel de concentración, lo que puede significar que la intensificación de los grados de especialización son en parte resultado del debilitamiento de las otras ciudades en este sector. 
Durante el periodo de estudio, las ciudades de Tula y Querétaro pasaron a estar especializadas en este tipo de industria. De esta manera, Querétaro se posicionó en poco tiempo como la segunda ciudad de mayor aumento en su participación del empleo de estos sectores, ante el proceso de desincorporación de esta industria del Valle de México. Así, tanto Toluca como Querétaro fueron ciudades que experimentaron una fuerte especialización en los sectores industriales de va bajo como resultado de la desindustrialización del Valle de México.

Figura 1. Sectores de especialización de las ciudades de la región centro en 2013

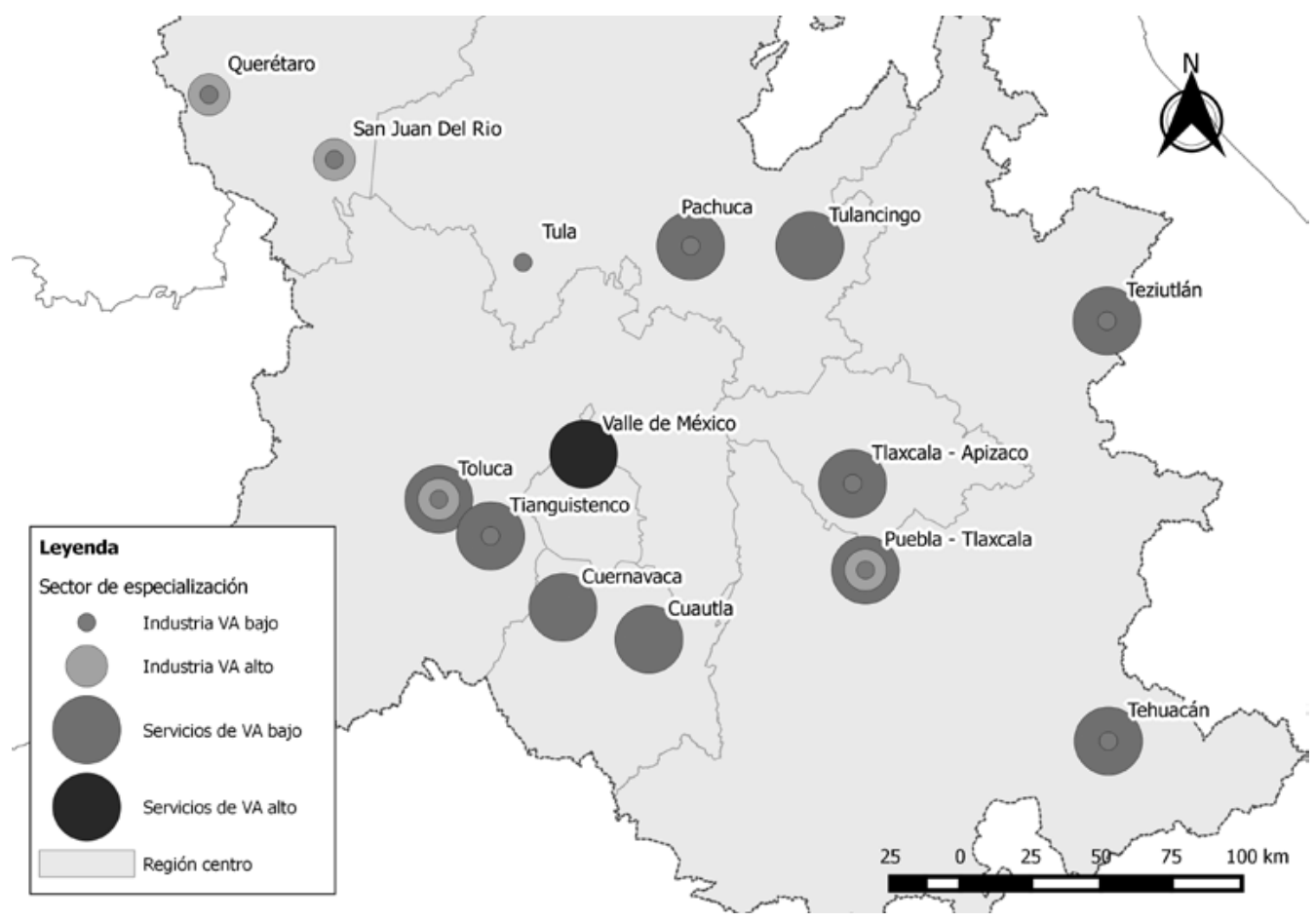

Fuente: elaboración propia con datos de INEGI (2014).

La tendencia entre especialización y tamaño poblacional demuestra que en las ciudades pequeñas se presenta una gran variabilidad de sus grados de especialización. La variabilidad comienza a ser menor en ciudades de tamaño medio y grande. Esto responde principalmente a que las ciudades pequeñas son más heterogéneas entre ellas, cada una con distintos atributos.

Por otra parte, las ciudades de mayor tamaño contienen en general una mayor cantidad de actividades y funciones que hacen que su empleo se encuentre distribuido entre varios sectores, lo cual puede disminuir su especialización en algunos sectores. 
La especialización de ciudades en sectores industriales de va alto muestra una tendencia opuesta a los de va bajo, pues se trata de ciudades de mayor tamaño. Querétaro, Toluca, Puebla-Tlaxcala y San Juan del Río se especializan en este tipo de sectores. Estas ciudades se encuentran localizadas cercanas a la capital del país, así como hacia el norte de la región, debido a que, al tratarse de industrias de alta tecnología, necesitan condiciones específicas para su desarrollo, así como vías de comunicación hacia el mercado exterior hacia las cadenas de valor de las que forman parte. No resulta extraño, por tanto, que las dos ciudades del estado de Querétaro sean las que reportaron un mayor grado de especialización en esta industria, y que fueron también las que incrementaron de manera importante su intensidad de 1998 a 2013, pues se trata de ciudades que conectan con la región del norte de México y con orientación hacia los Estados Unidos. Querétaro es la ciudad que se ha posicionado como la más vibrante en este sector y la de mayor crecimiento.

La tendencia observada en la especialización en industrias de va alto evidencia un proceso de distribución de sectores económicos de mayor importancia, tecnología y mano de obra calificada en centros urbanos más grandes y de mayor jerarquía regional. Los sectores industriales tradicionales han sido dispersados a ciudades de menor tamaño, mientras que los sectores modernos se han mantenido ubicados en las grandes ciudades. Es necesario tomar en consideración que Querétaro y San Juan del Río experimentan un comportamiento que se separa de la tendencia general, ya que las dos tienen altos grados de especialización en estos sectores, pero con un tamaño de población medio. Esto puede dar cuenta de la presencia de ventajas locacionales y competitivas de ambas ciudades, que han intensificado su actividad en estos sectores de 1998 a 2013.

En lo que concierne a los servicios de va bajo, se observa que la mayor parte de las ciudades de la región centro presentan especialización en estos sectores. Estas diez ciudades se localizan principalmente en la corona regional y el sur, son ciudades tanto de menor tamaño como ciudades grandes. Sin embargo, parte de las ciudades pequeñas son las que presentan los mayores grados de especialización, como Cuautla, Tulancingo y Pachuca. Al tratarse de servicios que requieren menor tecnología y mano de obra calificada, las ciudades pequeñas pueden tener la capacidad de proveerlos en sus poblaciones.

En los sectores de servicios de va bajo no se presenta una relación significativa entre el grado de especialización y el total de la población. Esto obedece a que, como se ha mencionado previamente, se trata de servicios que tienen una mayor facilidad para instalarse en cualquier tipo de territorio, además de que se trata de servicios orientados a la población. Lo que llama la atención es que se presenta una gran heterogeneidad de los grados de especialización en ciudades de tamaño de población similar.

En cuanto a los servicios de va alto se evidencia que hay una relación positiva entre el grado de especialización en estos sectores y el tamaño de población de las ciudades. 
A medida que aumenta el tamaño de población, su grado de especialización se incrementa. Este tipo de sectores es de vital importancia para el desarrollo regional, ya que representan actividades altamente dinámicas que comandan la economía regional y nacional. Todo esto está inmerso en el proceso creciente de terciarización económica a escala mundial, además de que generalmente estos sectores han tendido a concentrarse en las grandes capitales del mundo.

El estudio de la especialización de las ciudades de la región central muestra la organización territorial de la economía regional. La ciudad más importante, de la región y del país, que es el Valle de México, se especializa en servicios de va alto. Las ciudades de la corona regional se especializan principalmente en Servicios de va bajo. En el sur de la región se localizan ciudades más enfocadas en sectores de va bajo. Hacia el norte se ubican las ciudades predominantemente de sectores industriales, tanto de va alto como bajo.

La composición productiva de las ciudades se puede analizar también con base en su diversificación económica. De esta manera, se puede observar si una ciudad concentra su empleo en pocos o varios sectores económicos. Para la región central del país, se observa que las ciudades con mayor grado de diversificación económica se encuentran orientadas en el corredor centro-noroeste (figura 2). Las ciudades de Valle de México, Tula, San Juan del Río y Querétaro se posicionan como las de mayor diversificación. Estas ciudades albergan una gran variedad de actividades económicas y, en los últimos años, han experimentado una mayor concentración tanto de industrias como de servicios de va alto.

La localización de ciudades según su grado de diversificación muestra que las menos diversificadas se ubican en general en la periferia de la región, a excepción de la periferia noroeste. En la corona regional, las ciudades de Cuautla, Tianguistenco y Cuernavaca reportan una diversificación económica baja, mientras que las ciudades más importantes de la corona, que son Toluca y Puebla-Tlaxcala, experimentan niveles medios. Es necesario recalcar que el Valle de México y Tula tienen un grado de diversificación económica similar aun cuando en términos poblacionales son totalmente desiguales.

En los planteamientos teóricos de la diversificación económica se menciona que en general el tamaño de población tiene una relación positiva con el grado de diversificación. Esto se debe a que una mayor población representa un umbral de demanda necesario para la instalación de ciertos sectores. Por esta razón, también se establece teóricamente que las ciudades con grados de diversificación similares tienen en general un tamaño de población similar. Esta situación se cumple para las ciudades de la región centro, ya que las más grandes, en tendencia, son las que experimentan los mayores grados de diversificación. Es necesario hacer notar que se presenta un proceso de estabilización de la diversificación una vez que las ciudades alcanzan un tamaño considerable de población. 
Revista CIFE / ISSN: 0124-3551 e-ISSN: 2248-4914 / Bogotá-Colombia / Vol. 22 N.o 37 / julio-diciembre 2020 / pp. 81-102

Cabe resaltar que tres ciudades de tamaño medio (San Juan del Río, Querétaro y Tula) han podido desarrollar una gran cantidad de actividades, con lo que se presentan economías de urbanización sin tener necesariamente una gran cantidad de población.

Figura 2. Índice de diversificación económica por ciudad

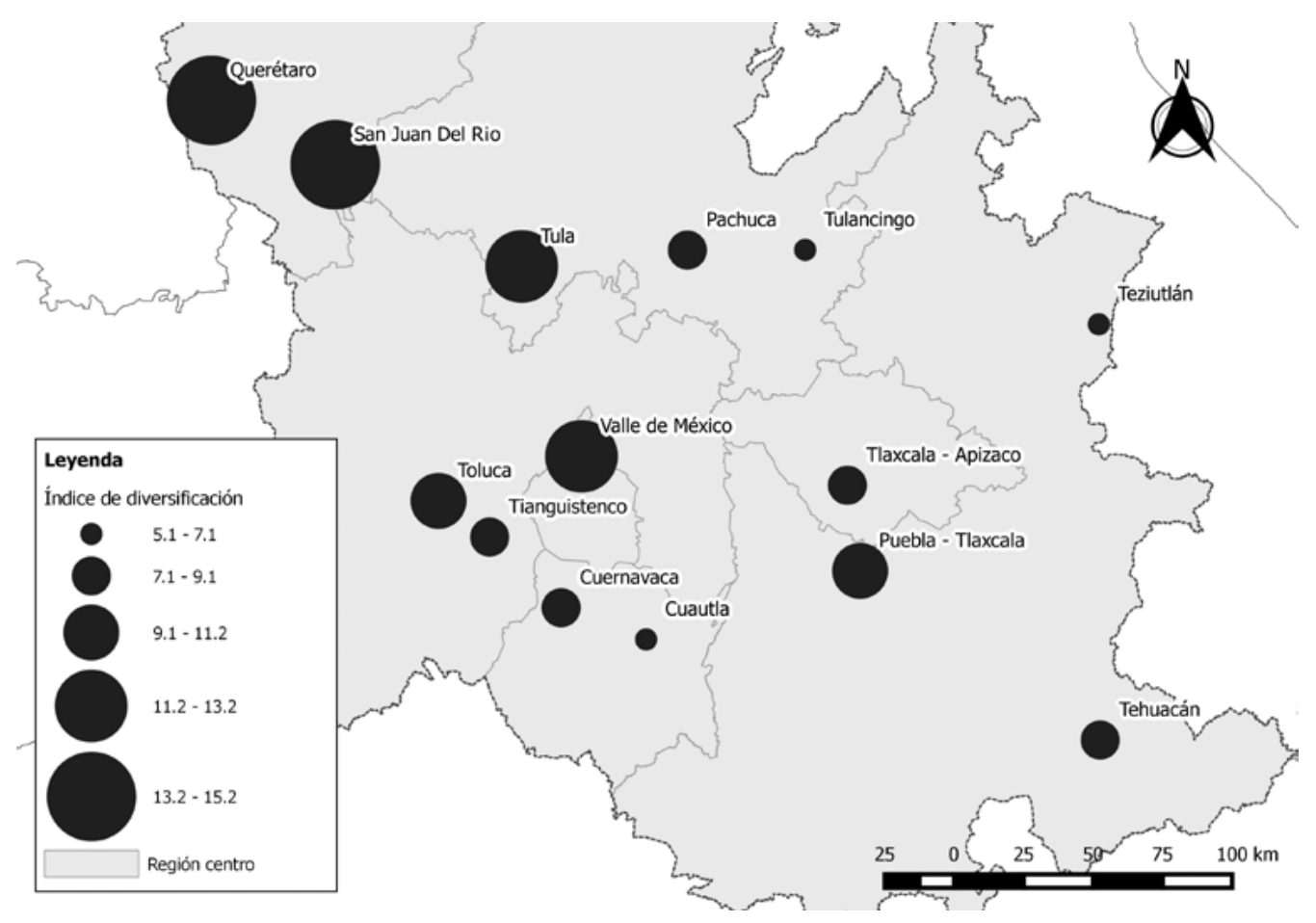

Fuente: elaboración propia con datos de INEGI (2014).

\section{Efectos en el crecimiento económico local}

El crecimiento económico de las ciudades es el motor principal del crecimiento regional y nacional, toda vez que en las zonas urbanas es donde se generan los procesos de crecimiento o declive de la economía. El crecimiento económico de las ciudades de la región ha sido heterogéneo (figura 3). La tendencia general muestra que de 1998 a 2013 el crecimiento fue poco dinámico sin grandes avances, debido a que a finales del siglo xx e inicios del xxI apenas se estaban empezando a reflejar los resultados de la apertura comercial. Para el periodo subsecuente, que va de 2003 a 2008, se experimentó el mayor ritmo de crecimiento. Empero este se vio seriamente afectado en el periodo 2008-2013, en el que las ciudades mostraron los ritmos de crecimiento más bajos de 
todo el periodo de estudio, principalmente por las repercusiones de la crisis inmobiliaria de los Estados Unidos, lo cual muestra el encadenamiento y dependencia de las ciudades con las economías internacionales.

Figura 3. Crecimiento económico de las ciudades de la región centro de 1998 a 2013

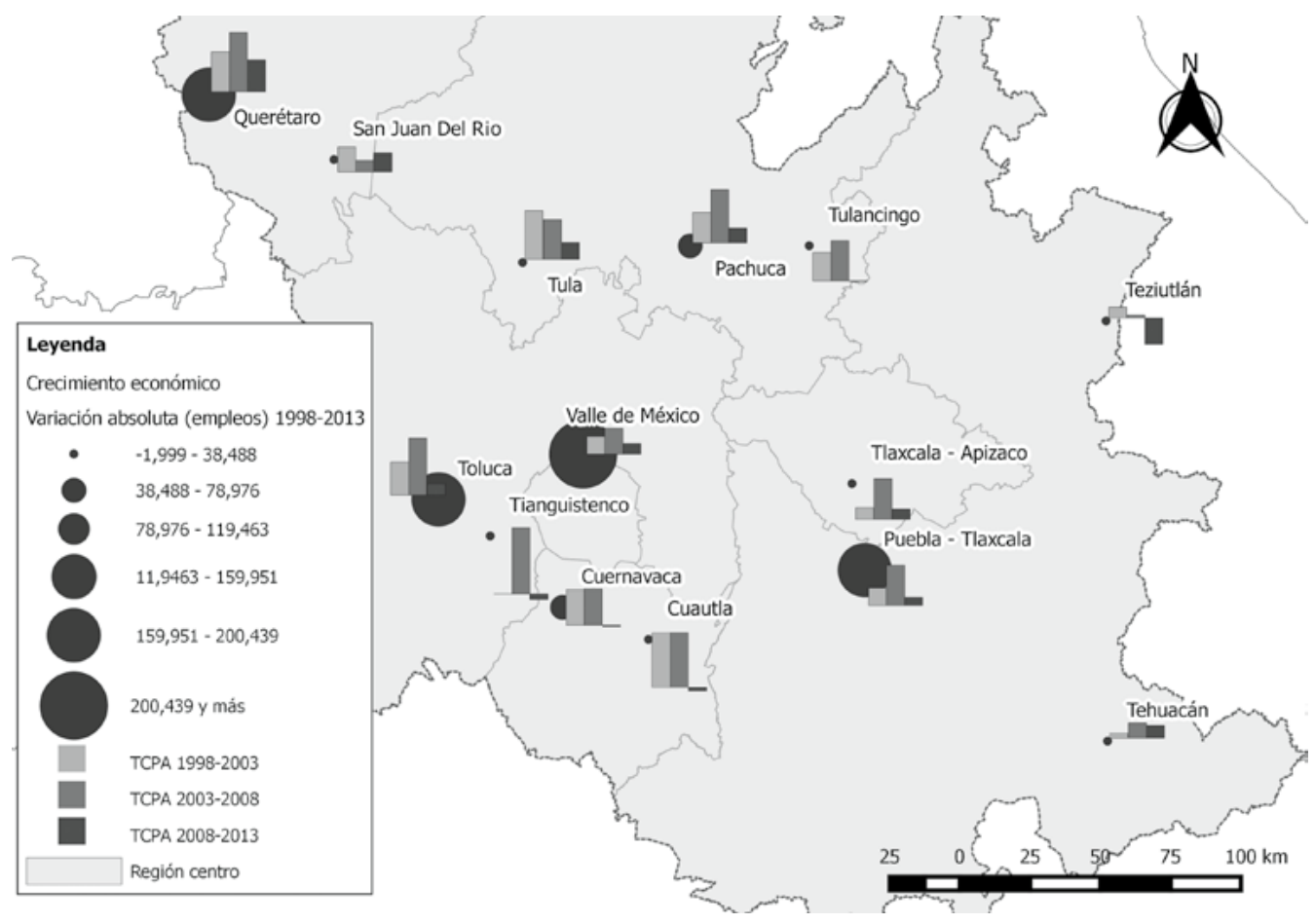

Fuente: elaboración propia con datos de INEGI (1999, 2014).

En el periodo de estudio, la ciudad con el mayor ritmo de crecimiento fue Querétaro, posicionándose como la ciudad más vibrante, mientras que la única que reportó un crecimiento negativo fue Teziutlán. El Valle de México creció a uno de los menores ritmos; no obstante, logró el mayor incremento de empleos en términos absolutos. El mayor ritmo de crecimiento, tanto en términos relativos como absolutos, se generó principalmente en una suerte de corredor que va desde Puebla-Tlaxcala hasta Querétaro.

La variación en los ritmos de crecimiento de las ciudades de la región se ven afectados por la estructura productiva de cada una de ellas. Esto se debe a los efectos de las economías de aglomeración, que podrían beneficiar a las ciudades según se encuentre especializada en algún sector o diversificada en varios. La especialización puede traer consigo economías de localización, mientras que la diversificación beneficiaría a las ciudades mediante las economías de urbanización. 
En cuanto a la presencia de economías de localización por especialización productiva, no se encontró un efecto fuerte de esta en el ritmo de crecimiento de las ciudades. De manera general, las ciudades que se encuentran especializadas en algún tipo de sector experimentaron tasas de crecimiento similares a las ciudades no especializadas (figura 4). Únicamente en el caso de las ciudades que se han especializado en industria de va alto, se puede observar que tuvieron un crecimiento ligeramente mayor a las ciudades no especializadas en esta industria; de esta manera, son el único sector en el que la especialización ha tenido un efecto positivo sobre el ritmo de crecimiento.

Figura 4. Gráficos de dispersión de la tasa de crecimiento promedio anual por tipo de especialización en 2013

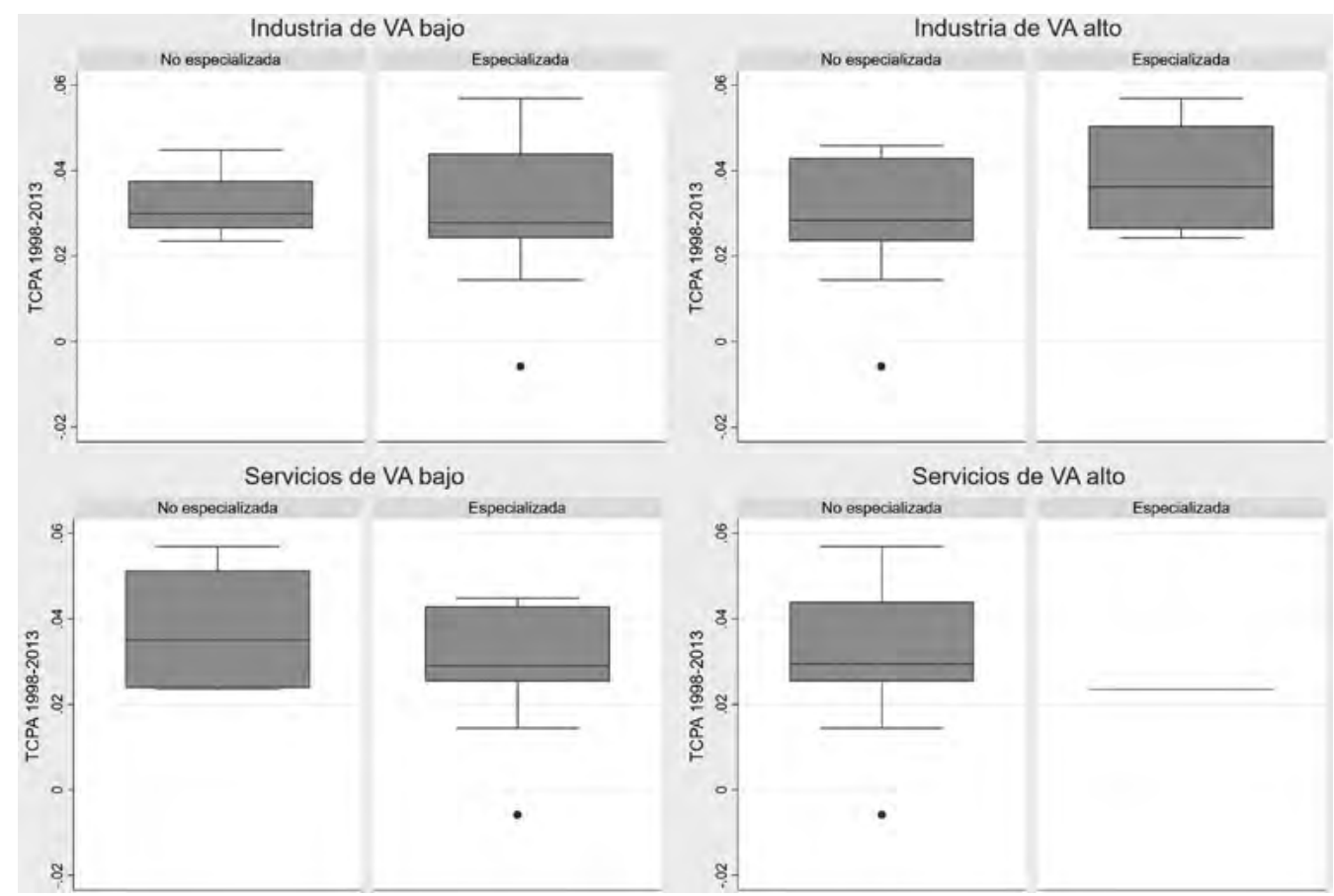

Fuente: elaboración propia con datos de INEGI (2014).

Los efectos de economías de localización en los sectores de servicios y la industria de vA bajo no cobran gran relevancia en el crecimiento. Cabe recalcar que el Valle de México es la única ciudad que resultó especializada en servicios de va alto y, si bien ha tenido una tasa de crecimiento relativamente baja, en realidad su crecimiento absoluto ha sido enorme. Por lo tanto, puede haber indicios de la influencia de las economías de localización en este tipo de sector para generar mayor crecimiento. 
En el caso de los efectos de la diversificación sobre el crecimiento, se encontró que esta ha tenido una importancia sustancial para incentivar el crecimiento económico local. Se evidencia que las economías de urbanización desempeñan un papel fundamental. Una estructura productiva más diversificada ha traído consigo un mayor ritmo de crecimiento. Las ciudades más diversificadas son las que más rápidamente han crecido en el periodo de estudio (figura 5).

Figura 5. Crecimiento económico e índice de diversificación por ciudad en 2013

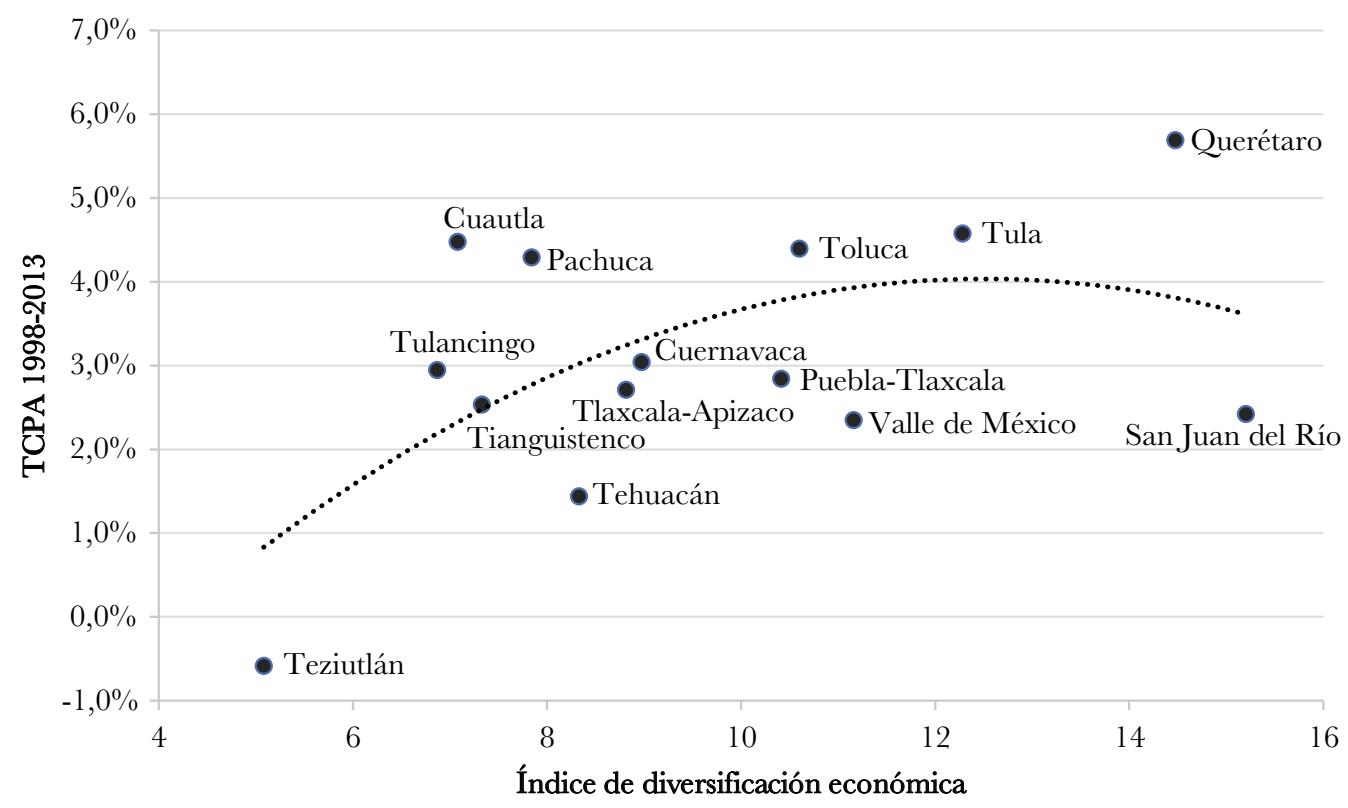

Fuente: elaboración propia con datos de INEGI (2014).

La influencia de la diversificación en el crecimiento puede dar evidencia de los beneficios de la aglomeración por efecto de la presencia de muchos sectores económicos. Esto ocasiona una posibilidad de mayor innovación y productividad en todos los sectores; además, se presenta una mayor disponibilidad de mano de obra y se cuenta con un mercado más grande con encadenamientos más fuertes. La tendencia muestra que, a medida que se incrementa el grado de diversificación, su efecto en el crecimiento económico es cada vez menor, es decir, experimenta rendimientos positivos pero decrecientes. Ello puede obedecer a que los beneficios de la diversificación se comienzan a difuminar a medida que se ha alcanzado cierto umbral de sectores económicos. De este modo, se puede establecer que las economías de urbanización tienen un límite para incentivar el ritmo de crecimiento de las ciudades. 


\section{Conclusiones}

El crecimiento económico local es uno de los fenómenos más complejos y variantes, máxime cuando se analizan las ciudades de la región más importante de México. En general, las ciudades en estudio mostraron dinámicas de crecimiento económico heterogéneo, que sobre todo se observó entre las ciudades de menor tamaño. La estructura productiva, ya sea que se trate de especialización o de diversificación económica, puede afectar de manera diferenciada a cada una de las ciudades.

Los reacomodos que se generan en la dinámica de distribución de actividades económicas entre ciudades han generado una mayor intensificación de la especialización sectorial en algunas ciudades y aumentado la diversificación en otras. Las ciudades con menor población se han enfocado principalmente en sectores de va bajo, tanto industriales como de servicios. Estas se localizan por toda la región, derivadas principalmente de la relocalización de sectores industriales de grandes a pequeñas ciudades, sobre todo aquellos sectores de baja calificación de la mano de obra. Por otra parte, las ciudades grandes se han especializado en sectores industriales y de servicios de va alto. Esto demuestra la tendencia general en la que los lugares centrales o ciudades principales son las que concentran la mayor parte de actividades estratégicas de vital importancia para la región.

La diversificación productiva, al menos en tendencia general, ha estado relacionada con la cantidad de población; las ciudades de mayor tamaño son también parte de las más diversificadas. No obstante, se presenta un fenómeno de un alto grado de diversificación económica sin necesariamente una gran cantidad de población, dado que ciudades pequeñas han podido desarrollar y albergar un gran número de sectores económicos. Esta situación puede estar fincada en las ventajas locacionales y competitivas de dichas ciudades. Cabe recalcar que la localización de las ciudades más diversificadas conforma una suerte de corredor desde el centro hasta el noroeste de la región. Esto puede estar asociado a la orientación de las exportaciones hacia la región norte del país, así como la cercanía con los Estados Unidos. Todo ello abre la posibilidad de poder contar con una estructura productiva más diversificada aún en ciudades medias o pequeñas, donde el tamaño de población ya no es una limitante.

El crecimiento económico de las ciudades de la región central de México fue acelerado hasta antes de la crisis inmobiliaria de 2008, tras la cual se experimentó el menor ritmo de crecimiento. De entre todas las ciudades, Querétaro se posicionó como la ciudad más dinámica de la región. Por su parte, el Valle de México, a pesar de que su tasa de crecimiento fue relativamente baja, continúa siendo la ciudad de mayor crecimiento en términos absolutos. Todo ello ha configurado y reforzado que la región central sea una región policéntrica, con una ciudad principal que continúa creciendo y un subcentro urbano que crece a ritmos acelerados. 
La estructura productiva de las ciudades ha sido uno de los principales factores para poder entender el ritmo de crecimiento de estas. En general, el crecimiento económico local está más relacionado con la composición productiva interna que con el tamaño de población. Se evidenció que hay una amplia heterogeneidad de crecimiento entre ciudades de tamaño equivalente, aunque se presenta una ligera tendencia a que las ciudades medias son las que crecen más. Los sectores de va bajo han mejorado el crecimiento de las ciudades más pequeñas, aunque con serias limitaciones. Por otra parte, la especialización en sectores de va alto ha beneficiado e impulsado a las ciudades con un mayor tamaño poblacional vía efectos de economías de localización, las cuales son más fuertes en estos sectores que en los de va bajo.

Uno de los mayores efectos de la estructura productiva en el crecimiento se origina en el seno de la diversificación económica. Las ciudades más diversificadas se han beneficiado de las economías de urbanización y han experimentado mayores ritmos de crecimiento. De esta manera, se ha demostrado que en la región central de México la fuerza de las economías de urbanización es más importante que las economías de localización.

Los resultados pueden ayudar a entender, con sus limitaciones, los factores que impulsan el crecimiento económico de las ciudades. Esto puede contribuir a generar políticas de desarrollo urbano y regional enfocadas en aprovechar los beneficios de la aglomeración sin descuidar la desigualdad urbana. Se trata, pues, de encontrar un equilibrio en el que el sistema urbano se beneficie de ciudades consolidadas que impulsen también el crecimiento de las ciudades en desarrollo, con el fin de fortalecer la región y el país. Sin embargo, aún quedan muchas tareas y temas pendientes. Si bien se realizó una comparación entre años, la falta de series de tiempo a escala de ciudades limita la incorporación de metodologías más sólidas como los modelos econométricos de series de tiempo. El número pequeño de ciudades en estudio restringió la posibilidad de hacer comparaciones más importantes entre distintos tipos de ciudades. Por esta razón, se requiere continuar analizando un fenómeno tan complejo como lo es el crecimiento económico local desde distintas aproximaciones teóricas y metodológicas.

\section{Referencias}

Aguilar, A. G. (1999). Mexico City Growth and Regional Dispersal: the Expansion of Largest Cities and New Spatial Forms. Habitat International, 23(3), 391-412. DOI: https://doi.org/10.1016/S0197-3975(99)00014-4

Aguilar, A. G. (2002). Megaurbanization and industrial relocation in Mexico's Central Region. Urban Geography, 23(7), 649-673. DOI: https://doi.org/10.2747/02723638.23.7.649 
Revista CIFE / ISSN: 0124-3551 e-ISSN: 2248-4914 / Bogotá-Colombia / Vol. 22 N.o 37 / julio-diciembre 2020 / pp. 81-102

Aguilar, A. G., \& Vásquez, M. I. (2000). Crecimiento urbano y especialización económica, en: México. Una caracterización regiones de las funciones dominantes. Investigaciones Geográficas, 42, 87-108. Recuperado de http://www.scielo.org.mx/pdf/ igeo/n42/n42a7.pdf

Álvarez, I., \& Guimon, J. (2010). Los sectores de alta tecnología en la Unión Europea. En Claves de la Economía Mundial. Madrid: Instituto de Comercio Exterior (Icex), Recuperado de https://www.researchgate.net/publication/274592712_Los_sectores_de_alta_tecnologia_en_la_Union_Europea

Asuad, N. (2012). Concentración Económica Espacial: Un enfoque de Dimensión Espacial de la Economía. Región Megalopolitana 1975-2003. En M. A. Mendoza, L. Quintana, N. Asuad (Coords.), Análisis espacial y regional: crecimiento, concentración, desarrollo y espacial (pp. 237-276). México, D. F.: Plaza y Valdés, S.A. y unam.

Balchin, P., Isaac, D., \& Chen, J. (2000). Urban economics. A global perspective. Nueva York: Palgrave.

Beeson, P. (1992). Agglomeration Economies and Productivity Growth. En E. Mills, J. McDonald, \& M. McLean, (Eds.), Sources of Metropolitan Growth (pp. 19-38). New Jersey: Rutgers, State University of New Jersey.

Bluestone, B., Stevenson, M., \& Williams, R. (2008). The urban experience. Nueva York: Oxford University Press.

Bostic, R., Gans, J., \& Stern, S. (1997). Urban productivity and factor growth in the late nineteenth century. Journal of Urban Economics, 41(1), 38-55. DOI: https://doi. org/10.1006/juec.1996.1087

Cingano, F., \& Schivardi, F. (2004). Identifying the sources of local productivity growth. Fournal of the European Economic Association, 2(4), 720-742. DOI: https://doi. org/10.1162/1542476041423322

Consejo Nacional de Población (Conapo). (2012). Catálogo del Sistema Urbano Nacional. México, D. F.: Conapo.

Dicken, P. (2011). Global shift: Mapping the changing contours of the world economy. London: SAGE Publications Ltd.

Dicken, P., \& Lloyd, P. (1990). Location in space: Theoretical perspectives in economic geography. New York: Harper Collins Publishers. 
Duranton, G., \& Puga, D. (2013). The growth of cities. En P. Aghion y S. N. Durlauf (Coords.), Handbook of Economic Growth (pp. 781-853), Volume 2. Elsevier. DOI: https:/ / doi.org/10.1016/B978-0-444-53540-5.00005-7

Fu, S., Dong, X., \& Chai, G. (2010). Industry specialization, diversification, churning, and unemployment in Chinese cities. China Economic Review, 21(4), 508-520. DOI: https://doi.org/10.1016/j.chieco.2010.04.007

Fujita, M., \& Thisse, J. (2013). Economics of agglomeration, cities, industrial location and globalization. New York: Cambridge University Press.

Garrocho, C. (2015). Dinámica de las ciudades de México en el siglo XXI: cinco vectores clave para el desarrollo sostenible. Ciudad de México: El Colegio Mexiquense.

Glaser, E., Kallal, H., Scheinkman, J., \& Shleifer, A. (1992). Growth in Cities. Journal of Political Economy, 100(6), 1126-1152. DOI: http://dx.doi.org/10.1086/261856

Glaeser, E. (2008). Cities, Agglomeration and Spatial Equilibrium. New York: Oxford University Press.

González, G., \& Peyrelongue, C. (1990). Querétaro: ciudades fragmentadas. Serie Ciudades. Dinámica urbano-regional en los noventa, 6. México: Red Nacional de Investigación Urbana.

Graizbord, B., \& Ruiz, C. (1999). Restructuración regional-sectorial en México, 19801993: una evaluación. Comercio Exterior, 49(4), 321-330. Recuperado de http://revistas.bancomext.gob.mx/rce/magazines/281/3/RCE3.pdf

Huallacháin, B. (1992). Economic Structure and Growth of Metropolitan Areas. En E. Mills, J. McDonald, \& M. McLean, (Eds.), Sources of Metropolitan Growth (pp. 51-85). New Jersey: Rutgers, State University of New Jersey.

Instituto Nacional de Estadística y Geografía (INEGI). (2014). Censos económicos 1999, 2004, 2009, 2014. México, D. F.: INEGI.

Jacobs, J. (1984). Cities and the Wealth of Nations: Principles of Economic Life. New York: Random House.

Krugman, P., \& Fujita, M. (2004). La nueva geografía económica: pasado, presente y futuro. Investigaciones regionales, 4(primavera), 177-206. Recuperado de https://www. redalyc.org/pdf/289/28900409.pdf

Marshall, A. (1890). Principles of Economics. Macmillan. 
Revista CIFE / ISSN: 0124-3551 e-ISSN: 2248-4914 / Bogotá-Colombia / Vol. 22 N.o 37 / julio-diciembre 2020 / pp. 81-102

McCann, P. (2013). Modern urban and regional economics. Oxford: Oxford University Press.

Mills, E., \& McDonald, J. (1992). Sources of Metropolitan Growth. New Jersey: Rutgers, State University of New Jersey.

Mokyr, J. (1990). The Lever of Riches: Technological Creativity and Economic Progress. New York: Oxford University Press.

Molero, J., \& Hidalgo, A. (2007). Los sectores de alta tecnología. En J. L. García-Delgado (Ed.), Estructura Económica de Madrid (pp. 501-530). Madrid: Thomson Civitas.

Nakamura, R., \& Morrison C. (2006). Measuring Agglomeration. En R. Capello \& P. Nijkamp (Eds.), Handbook of Regional Growth and Development Theories (pp. 305-328). Northampton: Edward Elgar Publishing.

Negrete, M. E. (2008). El centro de México: Evolución, limites y oportunidades para el desarrollo regional. Ciudad de México: Centro de Estudios Demográficos, Urbanos y Ambientales, El Colegio de México.

Quigley,J. (1998). Urban Diversity and Economic Growth. Fournal of Economic Perspectives, 12(2), 127-138. DOI: http://dx.doi.org/10.1257/jep.12.2.127

Richardson, H. (1978). Economía regional y urbana. Madrid: Alianza Editorial.

Sobrino, J. (2003). Competitividad de las ciudades en México. México, D. F.: El Colegio de México, Centro de Estudios Demográficos y de Desarrollo Urbano.

Valdivia, M. (2008). Desigualdad regional en el centro de México. Una exploración espacial de la productividad en el nivel municipal durante el periodo 1988-2003. Investigaciones Regionales, 13, 5-34. Recuperado de https://dialnet.unirioja.es/descarga/articulo/2756363.pdf 


\section{Anexo}

\section{Clasificación de sectores y subsectores según su valor agregado (VA)}

\section{Glasificación}

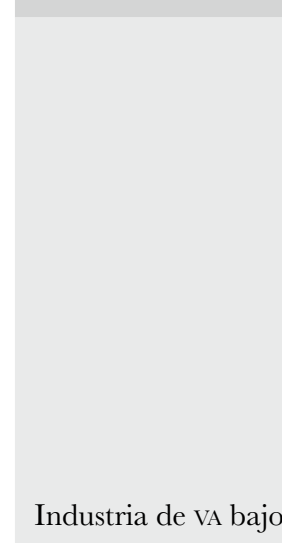

Industria de va alto

\section{Sector o subsector según clasificación INEGI}

314 Fabricación de productos textiles excepto prendas de vestir

321 Industria de la madera

315 Fabricación de prendas de vestir

337 Fabricación de muebles, colchones y persianas

316 Curtido y acabado de cuero y piel y fabricación de productos de cuero, piel y materiales sucedáneos

\section{Construcción}

\section{Impresión e industrias conexas}

311 Industria alimentaria

313 Fabricación de insumos textiles y acabado de textiles

332 Fabricación de productos metálicos

327 Fabricación de productos a base de minerales no metálicos

339 Otras industrias manufactureras

312 Industria de las bebidas y del tabaco

326 Industria del plástico y del hule

322 Industria del papel

335 Fabricación de accesorios, aparatos eléctricos y equipo de generación de energía eléctrica

334 Fabricación de equipo de computación, comunicación, medición y de otros equipos, componentes y accesorios electrónicos

336 Fabricación de equipo de transporte

333 Fabricación de maquinaria y equipo

331 Industrias metálicas básicas

325 Industria química

22 Generación, transmisión, y distribución de energía eléctrica, suministro de agua y de gas por ductos al consumidor final

324 Fabricación de productos derivados del petróleo y del carbón 


\section{Glasificación}

\section{Sector o subsector según clasificación INEGI}

81 Otros servicios excepto actividades gubernamentales

46 Comercio al por menor

72 Servicios de alojamiento temporal y de preparación de alimentos y bebidas

Servicios de va bajo 62 Servicios de salud y de asistencia social

53 Servicios inmobiliarios y de alquiler de bienes muebles e intangibles

71 Servicios de esparcimiento, culturales y deportivos y otros servicios recreativos

54 Servicios profesionales, científicos y técnicos

61 Servicios educativos

56 Servicios de apoyo a los negocios y manejo de desechos y servicios de remediación

Servicios de va alto

$$
43 \text { Comercio al por mayor }
$$

48-49 transportes, correos y almacenamiento

51 Información en medios masivos

52 Servicios financieros y de seguros

\section{Corporativos}

Fuente: elaboración propia con datos de INEGI (2014). 